\title{
DIAGNÓSTICO DE LA COMPETITIVIDAD AGROINDUSTRIAL EN EL DEPARTAMENTO DE BOYACÁ PARA EL AÑO 2011
}

\section{DIAGNOSIS OF AGRO-INDUSTRIAL COMPETITION IN THE STATE OF BOYACÁ IN 2011}

\section{Resumen}

El objetivo del presente artículo es identificar el estado de la competitividad agroindustrial en el departamento de Boyacá. Para ello se consideraron las variables de productividad y competitividad, junto con los factores de producción y mercadeo. Igualmente se tuvieron en cuenta las tecnologías de la información, la innovación y la gestión del conocimiento aplicado por las empresas. Para identificar los anteriores aspectos se construyó una encuesta, la cual fue aplicada a 106 empresas agroindustriales del departamento de Boyacá, durante el año 2011. Se concluyó que en términos generales el panorama actual del sector no es desfavorable; sin embargo, las empresas están descuidando elementos como el marketing, la inversión en innovación e investigación y en personal competente y la normalización de procesos; además, no se atrae conocimiento de las universidades y los recursos tecnológicos son muy limitados, lo que a mediano y largo plazo pone en serio peligro tanto el posicionamiento actual como la supervivencia del sector agroindustrial.

Palabras clave: competitividad, agroindustria, tecnología, conocimiento

\author{
Claudia Jessenia Becerra Gualdrón 1 \\ José Luis Cruz Vásquez 2 \\ Recibido: noviembre 12 de 2013
Aceptado: mayo 26 de 2014
}

\begin{abstract}
The purpose of this paper is to identify the state of agro-industrial competition in the State of Boyacá. To carry this out, the variables of productivity and competition were considered along with the factors of production and marketing. Also, technology, innovation and knowledge management applied by companies were taken into account. To identify the above features, a survey, which was administered to 106 agrobusinesses in the State of Boyacá, was applied in 2011. It was concluded that in general terms the current situation in the sector is not unfavorable; however, companies are not considering elements such as: marketing, investment in research, innovation and qualified personnel, and the standardization of processes. Also, expertise from universities is not usable and technological resources are very limited which in the middle and long term threatens both the current position and the survival of the agro-industrial sector.
\end{abstract}

Key words: competition, agro-industry, technology, knowledge 


\section{Introducción}

El término competitividad tiene un uso común y extendido en el mundo pero no existe consenso en su significado. Según Abdel y Romo (2004), la competitividad abarca diferentes niveles, a saber: empresa (nivel micro), país (nivel macro), industria y región (nivel meso). Para los fines de la presente investigación es pertinente el análisis de la competitividad desde el nivel industria, entendida esta última como el conjunto de empresas que se dedican a actividades económicas similares.

Según Porter (1990), la competitividad de una industria depende en gran medida de la competitividad nacional. Sala-i-Martin y otros (2012), afirman que factores como las instituciones, la infraestructura, las atenciones de salud y educación afectan la competitividad de las industrias de una nación. La competitividad de la industria depende de las empresas que la integran y del ambiente competitivo, generando círculos virtuosos entre una y otra, donde la industria competitiva presenta oportunidades para establecer eslabonamientos verticales, con efectos positivos en el desarrollo industrial del país (Abdel y Romo, 2004).

En este contexto, se puede entender la competitividad empresarial como la capacidad de una empresa de diferenciarse de otra. Esta diferenciación depende de elementos como las estrategias empresariales, la gestión de innovación (Esser, Hillebrand y Messner, 1999).

De otro lado, en el departamento de Boyacá la agroindustria ha sido identificada como un sector estratégico. Dentro de la Agenda Interna para la productividad y la competitividad de Boyacá (DNP, 2007), se identificaron cuatro apuestas productivas, entre las que se incluye la agroindustria.

En cuanto a la competitividad de la agroindustria boyacense, el Centro Regional de Gestión para la Productividad y la Innovación de Boyacá (CREPIB,
2010), ha publicado tres boletines virtuales que buscan identificar el estado de la productividad y competitividad de estos sectores en el departamento; de ellos se destacan elementos como: el bajo nivel de empresas certificadas con registro INVIMA, las dificultades en la comercialización fuera de Boyacá y la escasa implementación de gestión del conocimiento y desarrollo tecnológico, que conlleva niveles bajos de innovación.

A fin de fortalecer la agroindustria en el departamento de Boyacá, se requiere la identificación de los factores que determinan su competitividad. Por ello se adelantó un proyecto cuyos resultados se presentan a continuación. En la siguiente sección se presenta la metodología usada, la construcción del instrumento de medición y la selección de la muestra. En la tercera sección se exponen los resultados y se finaliza con las conclusiones y recomendaciones del estudio.

\section{Metodología}

Existe una discusión persistente tanto en el concepto de competitividad, como en los factores que la determinan. En el presente documento se entenderá a la competitividad como el grado en que un país, región o empresa produce bienes o servicios, enfrentando la competencia de los mercados nacionales o internacionales $y$ mejorando los ingresos reales de sus empleados y la productividad de sus empresas (Porter, 1990).

La discusión académica acerca de los factores que determinan la productividad es amplia; sin embargo, se ha llegado a ciertos niveles de consenso con factores como la productividad, la innovación, la tecnología, el conocimiento, la producción, el mercadeo y el talento humano.

El conocimiento es considerado el recurso intangible más valioso de las empresas (Augier y Teece, 2005; Teece, 1998; Tsai, 2001), además, la gestión y transferencia de conocimiento se han consolidado como elementos clave en el logro de 
una ventaja competitiva sostenible (Nonaka y Takeuch, 1995; Grant, 1996).

Para Porter (1990), la competitividad está determinada por la productividad. La productividad y la innovación son consideradas fundamentales para que una empresa logre ser competitiva (Lipovatz, Mandaraka y Mourelatos, 2000; Costa, Duch y Lladós, 2004) y la medición de estas variables es una aproximación para medir la competitividad (Echeverri y Escobar, 2004).

La incorporación de desarrollos tecnológicos a la producción es indudablemente una fuente de productividad e innovación que conlleva a mejorar la posición competitiva de las empresas (Conpes 3527, 2008; Cabrera, López y Ramírez, 2011).

De otro lado, el talento humano y la gestión de éste, han sido considerados determinantes de la competitividad de las empresas por cuanto un personal más y mejor preparado y con mayor participación dentro de las decisiones estratégicas de una organización puede impulsar su capacidad de inserción y permanencia en los mercados (Bonet, 1993; García y Espino, 2011; Fonnegra y González, 2006).

Teniendo en cuenta lo anterior, el marco de análisis para el presente documento se basa en las siguientes variables e indicadores:

\subsection{Indicadores de productividad}

Se utilizaron los indicadores del Manual Técnico de Estadísticas Estructurales e Indicadores Básicos de Productividad y Competitividad, Resolución 1261 de la Comunidad Andina de Naciones (2009):

\subsubsection{Valor bruto de la producción por establecimiento}

Es la cantidad del valor bruto de producción (VBP) por establecimiento (VBPe), mide la producción total del establecimiento industrial y se define como la producción de bienes y servicios para la venta y para la transferencia a otros establecimientos de la misma empresa, más la variación de existencias de productos en proceso, más el margen comercial por servicios prestados a terceros, certificados de reintegro tributario por ventas al exterior, a precios básicos, es decir sin los impuestos a los productos o los servicios. La unidad de medida es la moneda nacional y la definición operativa viene dada por la ecuación (1):

$V B P e=\frac{V B P}{\text { Número de establecimientos }}$

\subsubsection{Valor agregado promedio por establecimiento}

Es la cantidad del valor agregado (VA) por establecimiento (VAe). Constituye el incremento en el valor de los bienes y servicios que genera la actividad de explotación de la unidad de producción; es decir, es el valor creado por el propio establecimiento industrial. Se puede considerar también como el total de los ingresos recibidos por el uso de los factores productivos que participan en el proceso de producción durante el período de referencia. Se calcula como la diferencia entre el valor bruto de la producción, valorada a precios básicos y el consumo intermedio, valorado a precios de comprador y la unidad de medida es la moneda nacional. La definición operativa viene dada por la ecuación (2):

$V A e=\frac{\text { Valor agregado }}{\text { Número de establecimientos }}$

\subsubsection{Valor agregado respecto al valor bruto de producción}

Es la participación del valor agregado en el valor bruto de producción (VAvbp), en otras palabras, el porcentaje que representa el valor agregado en el 
valor bruto de producción. La unidad de medida es porcentaje y la definición operativa está dada por la ecuación (3):

VAvbp $=\frac{\text { Valor agregado }}{\text { Valor bruto de la producción }} * 100$

\subsubsection{Costo laboral promedio por establecimiento}

Es el valor monetario que devenga una unidad de mano de obra por su contribución a la producción de bienes y servicios por establecimiento. Permite medir el costo promedio de la mano de obra (sueldos y salarios, beneficios sociales y otras remuneraciones pagadas durante el año), que tiene el establecimiento. Se calcula tomando como información de referencia la sumatoria de sueldos y salarios básicos, aportes a seguridad social, otras remuneraciones, sobresueldos, subsidios, participación en utilidades y otros costos recibidos por el personal ocupado. La unidad de medida es la moneda nacional y la definición operativa está dada por la ecuación (4):

CLPe $=\frac{\frac{\text { Remuneraciones anuales }}{\text { Personal ocupado remunerado }}}{\text { Número de establecimientos }}$

\subsubsection{Productividad del trabajo por establecimiento}

La productividad laboral, se define como el cociente entre la producción real y la cantidad del insumo trabajo incorporado en el proceso de producción. Los cambios en la misma se definen como el cociente entre la variación de la producción y el cambio (aumento o disminución) de uso del factor trabajo. Este último puede ser medido de varias maneras, una de ellas es el número de empleados y las horas totales trabajadas, siendo ésta la más precisa, dado que mide el aporte promedio de cada persona ocupada, en la generación del valor agregado.

La productividad es el resultado que se obtiene por cada unidad de factor utilizado en la producción; está relacionada con la acumulación de capital físico y humano. Así, la productividad y, por extensión, la competitividad en el mercado interno, regional y mundial, depende de la acumulación de capital y el empleo de mano de obra para la producción. La unidad de medida es valores por unidad de personal ocupado y la definición operativa está dada por la ecuación (5), donde el valor agregado es la diferencia entre producción bruta y consumo intermedio.

$C L P e=\frac{\frac{\text { Valor agregado }}{\text { Personal ocupado }}}{\text { Número de establecimientos }}$

\subsubsection{Productividad del capital por establecimiento}

Es la contribución que realiza cada unidad monetaria de Activo Fijo a la constitución del Valor Agregado, por establecimiento. Mide el monto de productividad que genera el establecimiento por cada unidad monetaria de inversión en activos fijos. El valor de los Activos Fijos es igual a la sumatoria de: saldo inicial, construcciones por cuenta propia, compras de activos nuevos y usados, menos ventas o bajas. Se mide en porcentaje y la definición operativa está dada por la ecuación (6):

$P K e=\frac{\frac{\text { Valor agregado }}{\text { Activos fijos }}}{\text { Número de establecimientos }} * 100$

\subsubsection{Coeficiente técnico de rendimiento por establecimiento}

Es la cantidad de materias primas y bienes intermedios necesarios para la obtención de una unidad del producto. Los coeficientes técnicos sectoriales, miden las relaciones existentes entre los sectores económicos en función de la utilización de productos o inputs intermedios, que realiza una determinada rama sobre la producción final obtenida por dicha rama de actividad. El consumo intermedio es el valor a precios de 
comprador de todos los insumos utilizados por el establecimiento industrial durante el período de referencia. Considerando como insumos todos los bienes y servicios empleados en el proceso de fabricación o en la ejecución de los servicios que presta. Incluye: materias primas, combustibles, lubricantes, energía eléctrica, materiales auxiliares, envases, embalajes, repuestos, pagos por servicios industriales y otros. Se mide en porcentaje y la definición operativa viene dada por la ecuación (7):

$$
\text { CTe }=\frac{\frac{\text { Consumo Intermedio }}{\text { Valor bruto de la producción }}}{\text { Número de establecimientos }} * 100
$$

\subsubsection{Intensidad del capital por establecimiento}

Es la relación entre el valor de los activos fijos y el número de trabajadores, la unidad de medida es moneda nacional y la definición operativa está dada por la ecuación (8):

$$
I C e=\frac{\frac{\text { Activos fijos }}{\text { Número de trabajadores }}}{\text { Número de establecimientos }}
$$

\subsubsection{Personal ocupado promedio por establecimiento}

Es la cantidad de personal ocupado promedio contratado por establecimiento, la unidad de medida es número de personas y la definición operativa está dada por la ecuación (9):

$P O e=\frac{\text { Personal ocupado }}{\text { Número de establecimientos }}$

\subsection{Producción y Mercadeo}

Al analizar la competitividad de un sector es preciso indagar cómo y qué están produciendo las empresas y sus estrategias de mercadeo, a fin de conocer sus principales productos, a quién le está vendiendo sus productos, cuál es el uso de la capacidad instalada, cuáles son las principales materias primas que usa y de dónde las obtienen.

\subsection{Tecnologías, innovación y gestión del conocimiento}

Las tecnologías determinan el posicionamiento de una empresa en el mercado, esto implica conocer qué utiliza la empresa para transformar su producto, el uso de procesos estandarizados y la planeación del volumen de producción. A su vez, la permanencia en el mercado de un producto dependerá de las estrategias de mercadeo y de los mecanismos utilizados para darse a conocer.

De otro lado, existe un creciente consenso acerca de la importancia de la innovación en el aumento de la productividad y su relación directa con la competitividad. Por esto es preciso conocer las modificaciones realizadas a los productos, la forma de hacerlos y/o venderlos, los mecanismos de diferenciación, el uso de computadores e internet, la implementación y selección de nuevas tecnologías y la transmisión y protección del conocimiento.

\subsection{Instrumento de medición}

De acuerdo con la información anterior se elaboró una encuesta como instrumento de medición. Esta se dividió en cinco grandes partes: Identificación, Producción y Mercadeo, Innovación, Tecnología y Conocimiento, Talento humano e Información financiera.

El objetivo de la primera sección es conocer rasgos generales de la empresa: nombre, ubicación, NIT y contacto. Además, se pregunta si la empresa tiene Registro INVIMA o no y con qué entidades se ha relacionado, esto con el fin de saber con qué instituciones ha tenido relación la empresa en su tiempo de existencia.

La segunda sección pretende saber cómo y qué están produciendo las empresas y algunas estrategias de mercadeo. De este modo, se indagó 
sobre los principales productos, a quién se los están vendiendo, cuál es el nivel de uso de la capacidad instalada, las principales materias primas usadas y dónde las obtienen. Del mismo modo, se pregunta sobre las tecnologías que utiliza actualmente la empresa para transformar su producto, si tiene procesos estandarizados y cómo realiza la planeación del volumen de producción. También se indaga por los mecanismos utilizados para darse a conocer.

En la tercera sección: innovación, tecnología y conocimiento, se indaga acerca de las modificaciones realizadas a los productos, la forma de hacerlos y/o venderlos, los mecanismos de diferenciación usados y los que según el punto de vista de los empresarios son los mayores obstáculos para mejorar la productividad, competitividad e innovación de la empresa. A su vez, se incluyen preguntas acerca del uso de computadores e internet, así como la implementación y selección de nuevas tecnologías y la transmisión y protección del conocimiento.

La cuarta sección está relacionada con el Talento Humano; aquí se trata de establecer el número total de trabajadores, el género, el tipo de vinculación y el nivel de estudio. Finalmente, se solicita la información financiera de la empresa con el objetivo de calcular los indicadores de productividad. Dentro de los datos requeridos se encuentran, costos, ingresos y activos.

\subsection{Población y muestra}

En primer lugar se procedió a acceder a las bases de datos sobre empresas agroindustriales del departamento de las Cámaras de Comercio de Boyacá del año 2011. Tras la revisión y depuración de dichas bases de datos, se obtuvo un tamaño poblacional de 658 elementos. De los cuales el $19 \%$ corresponden a la jurisdicción de la Cámara de Comercio de Sogamoso, el 36\% a la de Duitama y el restante $45 \%$ a la de Tunja.
El tamaño muestral se calculó con $90 \%$ de confianza, $7.35 \%$ de margen de error y varianza máxima; con base en la ecuación (10) (Martínez, 2012):

$n=\frac{N^{*} Z_{\alpha}^{2} p^{*} q}{d^{2} *(N-1)+Z_{\alpha}^{2} * p^{*} q}$

El resultado fue de 106 elementos. La metodología de recolección de información fue la aplicación personal de la encuesta al propietario o gerente de la empresa, asimismo y con el fin de obtener consistencia y uniformidad en la interpretación de las preguntas se diseñó un protocolo de aplicación.

\section{Resultados y discusión}

\subsection{Subsectores y ubicación de la población de empresas agroindustriales de Boyacá}

Del total de población de las empresas agroindustriales de Boyacá (658), se encontró que los subsectores de panadería y lácteos son los que cuentan con el mayor número de establecimientos, tal como se observa en la figura 1. Dentro de los productos de panadería se encuentra la elaboración de amasijos y comidas típicas y dentro de otros productos alimenticios la fabricación de bocadillos. Duitama, Sogamoso y Tunja, tienen el mayor número de establecimientos dedicados a la agroindustria en Boyacá, de acuerdo con la figura 2. Los municipios incluidos en la figura son los que cuentan con una participación igual o superior al $1 \%$ de los establecimientos.

\subsection{Información general empresas encuestadas}

De acuerdo con el número de empleados, la gran mayoría de establecimientos agroindustriales en Boyacá son microempresas (86\%), le siguen mediana (14\%) y gran empresa (1\%). Además, el promedio de permanencia en el mercado es de 9.4 años. 


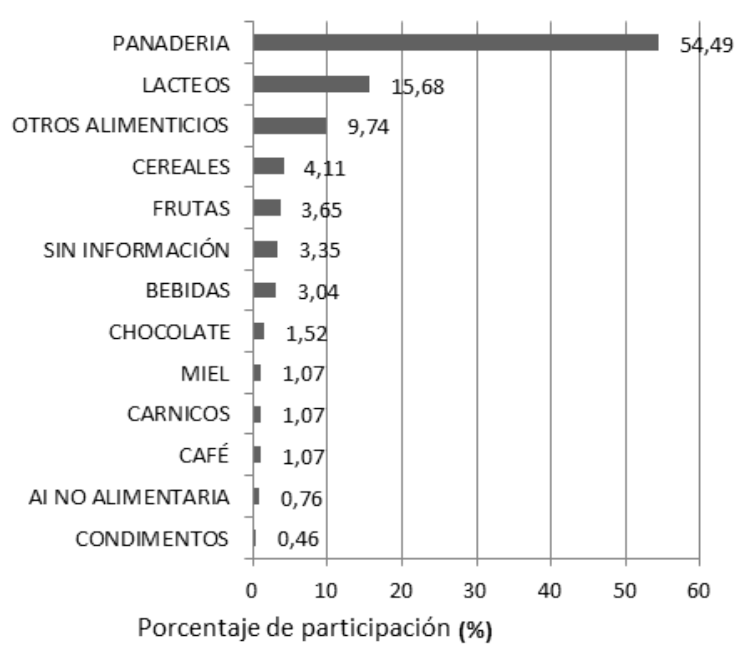

Figura 1. Subsectores de la agroindustria en Boyacá por porcentaje de participación

El $47.2 \%$ de las empresas no cuentan con registro INVIMA, cifra similar a la presentada en el año 2010, lo cual indica que las empresas agroindustriales de Boyacá no han evolucionado mucho en este aspecto y aún tienen dificultades para garantizar trazabilidad en los bienes. Teniendo en cuenta que el $85 \%$ de los colombianos consideran muy importante este registro (Franco, 2009), esto revela una dificultad para el posicionamiento de las empresas agroindustriales en diversos mercados.

En el ambiente actual, el uso de las tecnologías de la información y las comunicaciones es fundamental para la expansión y fortalecimiento de una unidad productiva. En este sentido, el 23\% de las empresas encuestadas tienen teléfono fijo, mientras que el $66 \%$ tienen celular, el $33 \%$ tienen correo electrónico y tan sólo el $4.72 \%$ tienen página web. El $40 \%$ usan computadores dentro de la empresa y sólo el $28 \%$ usan internet, en su gran mayoría para fines contables (26.4\%), así como para acceder a información específica $(9,4 \%)$, correo electrónico $(5.7 \%)$, acceso a información general (5.7\%), trámites con el estado (3.8\%) y en menor medida publicidad (2.58\%) y ventas (1.9\%).

Las entidades con las que más se relacionan las

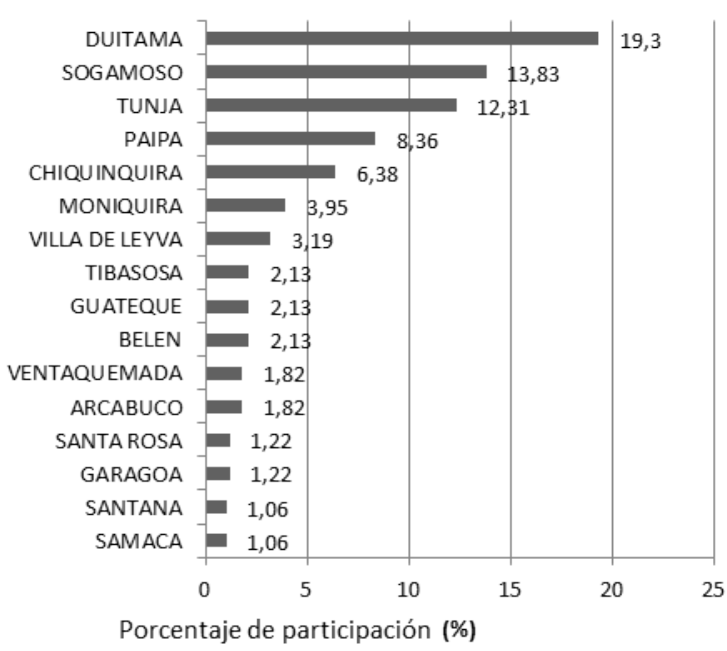

Figura 2. Municipios de la agroindustria en Boyacá por porcentaje de participación

empresas son las Cámaras de Comercio del departamento (74.5\%), el SENA (11.3\%), la Gobernación de Boyacá (2.8\%) y el CREPIB (0.9). Entre otras entidades se encuentran incubadoras, proveedores, Fondo Emprender, Acopi, Universidad Santo Tomás, Ministerio de Agricultura y Transporte.

\subsection{Indicadores de productividad de la agroindustria de Boyacá 2011}

Según se observa en la tabla 1, el precio promedio de los productos agroindustriales disminuyó $2.79 \%$ entre el primer y segundo trimestre de 2011, pero aumentó 3.04\% durante el tercer trimestre. Asimismo para el tercer trimestre de 2011, el promedio de uso de la capacidad instalada fue del $87 \%$, registrando un aumento de $0.68 \%$.

El Valor bruto de la producción por establecimiento, presentó una disminución durante el segundo trimestre del año, pero volvió a crecer para el tercero. Por su parte, el Valor agregado promedio por establecimiento de las empresas agroindustriales superó los 35 millones de pesos, pero bajó durante el segundo trimestre $y$, al igual que el valor bruto de producción aumentó en el tercero. El valor agregado respecto 
DIAGNÓSTICO DE LA COMPETITIVIDAD AGROINDUSTRIAL EN EL DEPARTAMENTO DE BOYACÁ PARA EL AÑO 2011

\begin{tabular}{|c|c|c|c|}
\hline Nombre del indicador & $\begin{array}{c}\text { Primer } \\
\text { trimestre } 2011\end{array}$ & $\begin{array}{c}\text { Segundo trimestre } \\
2011\end{array}$ & $\begin{array}{c}\text { Tercer } \\
\text { trimestre } 2011\end{array}$ \\
\hline Valor bruto de la producción por establecimiento $\$$ & $65.741 .733,33$ & $65.434 .000,00$ & $66.411 .973,68$ \\
\hline Valor agregado promedio por establecimiento $\$$ & $35.808 .356,16$ & $35.371 .301,37$ & $35.801 .824,32$ \\
\hline Valor agregado respecto al valor bruto de producción (VAvbp) \% & 48.88 & 48.31 & 48.96 \\
\hline Costo laboral promedio por establecimiento $\$$ & $1.133 .453,91$ & $1.116 .932,90$ & $1.147 .316,85$ \\
\hline Productividad del trabajo por establecimiento (PT) \$ & $3.206 .170,57$ & $3.116 .418,93$ & $3.378 .457,19$ \\
\hline Productividad del capital por establecimiento (PK) \% & 10.19 & 8.80 & 10.03 \\
\hline Coeficiente técnico de rendimiento por establecimiento (CT) \% & 55.10 & 55.67 & 54.98 \\
\hline Intensidad del capital por establecimiento $\$$ & $11.940 .043,13$ & $12.316 .080,30$ & 11.223.341,17 \\
\hline Personal ocupado promedio por establecimiento (Número de personas) & 9.10 & 9.14 & 9.08 \\
\hline
\end{tabular}

Tabla 1. Indicadores de Productividad y Competitividad Agroindustrial DE Boyacá para el año 2011

al valor bruto de producción que representa el porcentaje de valor agregado dentro del valor bruto de la producción, fue de $48.88 \%$ durante el primer trimestre con comportamiento similar al de los indicadores anteriores.

En cuanto al costo laboral promedio por establecimiento y la productividad laboral o productividad del trabajo, se tiene que el primer indicador supera el millón de pesos y el segundo los 3 millones de pesos. La productividad del capital respecto a la inversión en activos fijos fue de $10.19 \%$ y disminuyó en los trimestres siguientes. El coeficiente técnico de rendimiento por establecimiento fue de $54.98 \%$ para el tercer trimestre, es decir, que se requiere esta cantidad de insumos para generar una unidad de producción de bienes y servicios. La intensidad del capital definida como la relación entre los activos fijos y el número de trabajadores estuvo cerca a los 12 millones de pesos y finalmente, las empresas tuvieron en promedio 9 empleados.

\subsection{Producción y mercadeo de la agroindustria de Boyacá 2011}

El $92 \%$ de los entrevistados ofrecen bienes de consumo final, mientras que el $8 \%$ se constituyen en productos intermedios. Según se observa en la figura 3 , el $77.4 \%$ de las empresas venden en su ciudad de ubicación, el $30,2 \%$ a otros establecimientos como colegios y alcaldías y el $13.2 \%$ venden a Bogotá. El $53 \%$ de las empresas tiene algún tipo de proceso estandarizado, de las cuales, la mayoría son procesos de empaque y envoltura (27.4\%) seguidos de sistemas de calidad (1.9\%).

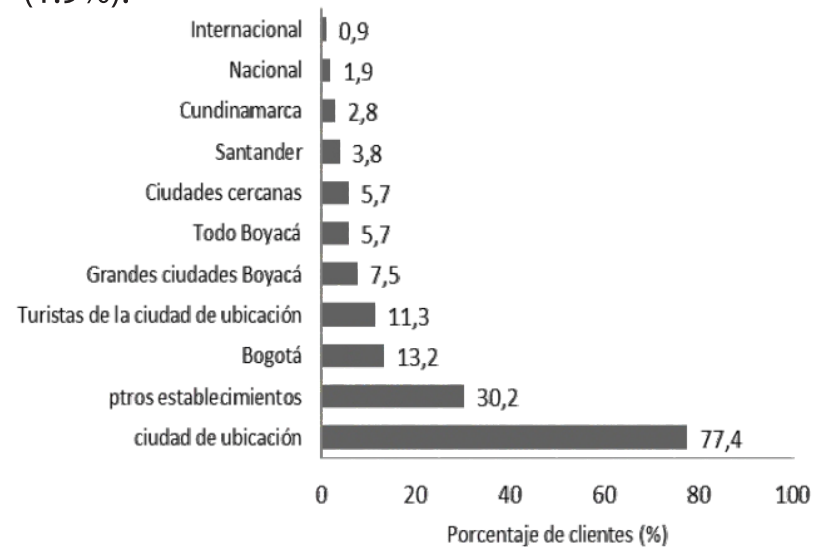

Figura 3. Ubicación de los clientes

La planeación de la producción, se realiza en su mayoría teniendo en cuenta las ventas anteriores (72\%), de igual forma se realiza por pedidos (18\%), teniendo en cuenta la disponibilidad de la materia prima (10\%) y finalmente por pronóstico de ventas (8\%). Asimismo el $67 \%$ de las empresas cuenta con 
logotipo para identificarse, esta cifra es menor a la registrada en el 2010 (75\%). A pesar de la importancia de tener un símbolo distintivo muchas empresas no cuentan con este medio de publicidad. Del mismo modo, sólo el $68 \%$ de los establecimientos realiza algún tipo de publicidad, los medios usados se presentan en la tabla 2.

\begin{tabular}{lc}
\hline \multicolumn{1}{c}{ Medio de publicidad } & Empresas (\%) \\
\hline Cliente a cliente & 66.0 \\
Radio & 20.8 \\
Afiches, vallas & 8.5 \\
Tarjetas & 7.5 \\
Etiquetas, punto de venta & 7.5 \\
Volantes & 6.6 \\
Periódico, directorio & 5.7 \\
Internet & 2.8 \\
Televisión & 1.9 \\
Eventos & 1.9 \\
\hline
\end{tabular}

Tabla 2. Porcentaje de empresas por medio de publicidad

\subsection{Innovación, desarrollo tecnológico y gestión del conocimiento de la agroindustria de Boyacá 2011}

El $73 \%$ de los empresarios consideran que su principal diferenciación es la calidad, seguido del precio y el sabor. Esto se puede interpretar como que los empresarios boyacenses están conscientes que para llegar a nuevos mercados y consolidarse en los que ya tienen es fundamental cumplir con las expectativas de los clientes en términos de calidad. No obstante, la innovación como medio diferenciador, es usada sólo por el $6.6 \%$ de las empresas. Otros factores de diferenciación son el precio (17.08\%), el sabor $(13.2 \%)$, la atención (12.3\%), la presentación (8.5\%) y en menor medida la tradición (1.9\%).

Por otra parte, el $46 \%$ de las empresas ha implementado algún tipo de innovación incremental, de los cuales el $30.2 \%$ ha sido en marketing, el $27.4 \%$ en proceso, el $19.8 \%$ en producto y el $7.5 \%$ en organización.
La tabla 3, señala los que a juicio de los empresarios son los mayores obstáculos para mejorar la productividad, competitividad e innovación de sus empresas. El acceso al sistema financiero y la escasez de recursos económicos son señalados por las empresas como los principales causantes de los rezagos en productividad, competitividad e innovación, seguidos de la disponibilidad y/o precios de materias primas o insumos.

\begin{tabular}{lc}
\hline $\begin{array}{l}\text { Mayores obstáculos para mejorar la } \\
\text { productividad, competitividad e } \\
\text { innovación de la empresa }\end{array}$ & Porcentaje (\%) \\
\hline $\begin{array}{l}\text { Falta de recursos económicos/Acceso } \\
\text { sistema financiero }\end{array}$ & 34.0 \\
$\begin{array}{l}\text { Oferta/Precios de materias } \\
\text { primas/insumos }\end{array}$ & 18.9 \\
Información, Capacitaciones, Tecnologías, & 17.0 \\
Innovación & \\
Alta competencia/Competencia desleal & 14.2 \\
Falta estrategias Marketing & 12.3 \\
Falta de apoyo institucional & 11.3 \\
Altos impuestos & 4.7 \\
Condiciones climáticas & 3.8 \\
Altos precios de servicios públicos & 2.8 \\
Altos requisitos normatividad & 2.8 \\
Mal estado vías de comunicación & 1.9 \\
\hline
\end{tabular}

Tabla 3. Obstáculos para mejorar la productividad, competitividad e innovación de la empresa

Asimismo, el 35\% de las empresas han adquirido e implementado nuevas tecnologías; llama la atención que el $40 \%$ lo hizo hace menos de un año, el $42.9 \%$ entre 1 y 3 años, el $14.3 \%$ entre 4 y 6 años y el $2.9 \%$ lo hizo hace más de seis años.

La selección de tecnologías se hace de acuerdo a la tabla 4, así, los requerimientos en normatividad y las necesidades empresariales son los criterios usados por mayor número de empresas.

El $22.6 \%$ de las empresas no tiene ningún método de transmisión de conocimiento, en las que sí lo tienen se destaca el aprendizaje en la práctica (42.5\%), las capacitaciones y conferencias (34\%) y en menor porcentaje, contratación de expertos, 


\begin{tabular}{lc}
\hline Criterios de selección de tecnologías & Porcentaje (\%) \\
\hline Necesidad empresa/Normatividad & 17.9 \\
Mejora de la productividad & 10.4 \\
Asesoría/Expertos & 5.7 \\
Mejora de calidad & 2.8 \\
Reducción costos & 1.9 \\
Necesidad mercado & 1.9 \\
Normalizar procesos & 0.9 \\
Depreciación & 0.9 \\
Bienestar empleados & 0.9 \\
Seguridad & 0.9 \\
\hline
\end{tabular}

Tabla 4. Criterios de selección de tecnologías por porcentaje de empresas

periodos de prueba y documentos. En este mismo sentido, casi el $51 \%$ de las empresas no realizan ningún tipo de protección del conocimiento, en su mayoría porque se trata de conocimiento genérico, no obstante el $27.4 \%$ realiza acuerdos de confidencialidad, de igual forma realizan un trabajo riguroso de selección del personal de modo que sea de confianza (14.2\%), el $9.4 \%$ mantiene secretos industriales y solo el $0.9 \%$ realiza registro de patentes.

\subsection{Análisis de Debilidades, Oportunidades, Fortalezas y Amenazas (DOFA)}

Teniendo en cuenta la información anterior y la Agenda Interna para la productividad y la competitividad de Boyacá (2007), se construyó la siguiente matriz de Debilidades, Oportunidades, Fortalezas y Amenazas, DOFA, con el objetivo de evaluar el estado de las empresas agroindustriales del departamento y tener un insumo para proponer alternativas de mejora.

A partir del análisis DOFA se pueden proponer las siguientes estrategias:

Establecer canales de comunicación y comercialización de materias primas y salida de productos.

Incorporar paulatinamente mejoras (innovaciones) en la producción que impliquen mayor generación de valor, bien sea por cuenta propia o por medio de convocatorias públicas o privadas.

Incorporar tecnologías para la conservación y transporte apropiado de las materias primas, aprovechando la calidad de las tierras y la cercanía de las materias primas.

Establecer estrategias de marketing al interior de la empresa para aprovechar la cercanía al principal mercado del país y el reconocimiento de los consumidores de los productos de la región

Realizar talleres para la construcción de confianza y campañas de culturización acerca de la importancia de la asociatividad dentro de los empresarios, para aprovechar la gran cantidad de micro y pequeñas empresas presentes en el departamento.

Estrechar las relaciones con entidades presentes en la región como universidades, SENA, institutos técnicos, CREPIB y Centros de Investigación para aprovechar la alta oferta de personal calificado, además, para la incorporación de investigación y desarrollo dentro de la empresa.

Formalizar las unidades productivas para aprovechar los incentivos legales.

Realizar alianzas entre las micro y pequeñas empresas para establecer grupos de presión en mejores posiciones de negociación ante instituciones gubernamentales y financieras, entre otras.

Implementar técnicas de gestión del conocimiento para evitar la pérdida de conocimiento valioso de los productos y procesos de las empresas. 


\begin{tabular}{|c|c|}
\hline FORTALEZA & DEBILIDAD \\
\hline $\begin{array}{ll}\text { - } & \text { Tradición productiva y saber-hacer local. } \\
\text { - } & \text { Concentración de las fábricas. } \\
\text { - } & \text { Calidad en los productos. } \\
\text { - } & \text { Calidad de las tierras. } \\
\text { - } & \text { Seguridad en el campo. }\end{array}$ & $\begin{array}{l}\text { - } \quad \text { Poca sofisticación y baja agregación de valor en los procesos productivos. } \\
\text { - } \quad \text { Baja productividad y capacidad de generación de empleo en los sectores } \\
\text { - } \quad \text { Altos niveles de informalidad empresarial y laboral. } \\
\text { - } \quad \text { Bajos niveles de innovación y de absorción de tecnologías. } \\
\text { - } \quad \text { Estacionalidad de las cosechas. } \\
\text { - } \quad \text { Problemas de certificación de calidad, casi la mitad de las empresas no tienen } \\
\text { INVIMA. } \\
\text { - } \quad \text { Problemas en la estandarización de procesos. } \\
\text { - } \quad \text { Bajo uso de TIC's. } \\
\text { - } \quad \text { Bajo relacionamiento con otras entidades. } \\
\text { - } \quad \text { Ventas concentradas en la ciudad de ubicación. } \\
\text { - } \quad \text { Problemas de marketing: débil imagen empresarial. } \\
\text { - } \quad \text { Escasa cultura de asociatividad. } \\
\text { - } \quad \text { Acceso limitado a capacitaciones e información sobre tendencias del sector. } \\
\text { - } \quad \text { Dificultades para la gestión del conocimiento. } \\
\text { - } \quad \text { Escasa protección del conocimiento generado dentro de la empresa. } \\
\text { - } \quad \text { Debilidad de la institucionalidad relacionada con la competitividad. }\end{array}$ \\
\hline OPORTUNIDAD & AMENAZA \\
\hline $\begin{array}{l}\text { - } \quad \text { Cercanía al principal mercado del país. } \\
\text { - } \quad \text { Concentración de la población en el sector. } \\
\text { - } \quad \text { Alta oferta educativa profesional y técnica. } \\
\text { - } \quad \text { Posibilidades de apertura de nuevos } \\
\text { mercados a nivel nacional y externo. } \\
\text { - Distribución de empresas en todo el } \\
\text { departamento. } \\
\text { - Cercanía de materias primas. } \\
\text { - Reconocimiento de los consumidores de } \\
\text { los productos de la región. }\end{array}$ & $\begin{array}{l}\text { - Escaso acceso al sistema financiero } \\
\text { - Deficiencias en la infraestructura de transporte y energía. } \\
\text { - Baja calidad y poca pertinencia de la educación. } \\
\text { - Estructura tributaria poco amigable a la competitividad. } \\
\text { - Rezago en penetración de tecnologías de información y en conectividad. } \\
\text { - Degradación ambiental como limitante de la competitividad. } \\
\text { - Altos precios de servicios públicos. } \\
\text { - Altos precios de bienes de capital } \\
\text { - Dependencia tecnológica. }\end{array}$ \\
\hline
\end{tabular}

Tabla 5. Matriz DOFA

\section{Conclusiones}

La información recolectada permitió la priorización de cinco factores positivos y cinco negativos que inciden sobre la competitividad de la agroindustria. Los positivos son la tradición productiva y saber-hacer local, la cercanía al principal mercado del país, la calidad de las tierras, el reconocimiento de los consumidores hacia los productos de la región y su calidad. Por su parte los factores negativos son la poca sofisticación y baja agregación de valor en los procesos productivos, los problemas de estandarización de procesos, apoyo institucional y acceso al sistema financiero, la baja productividad y capacidad de generación de empleo en los sectores formales y la escasa cultura de asociatividad.

El análisis DOFA permitió plantear estrategias que aporten al mejoramiento de la competitividad del sector agroindustrial de Boyacá, éstas giran alrededor de alcanzar normalización técnica en la producción y a partir de allí, implementar mejoras que se traduzcan en innovaciones con base en los requerimientos del mercado y las mejoras en las técnicas de producción. Del mismo modo, es fundamental la gestión de nuevos mercados y el aprovechamiento de la cercanía con Bogotá, así como el trabajo en el incentivo de la asociatividad.

En términos generales el panorama actual del sector no es desfavorable. Sin embargo, las empresas están descuidando elementos como el marketing, la inversión en innovación e investigación y en personal competente y la normalización de procesos; tampoco se atrae conocimiento de las universidades y los recursos tecnológicos son muy limitados, lo que a mediano 
y largo plazo pone en serio peligro tanto el posicionamiento actual como la supervivencia de las empresas.

\section{Agradecimientos}

El presente trabajo es resultado del proyecto de investigación: Determinantes de la competitividad de la agroindustria y el turismo en Boyacá, financiado por la Dirección de Investigaciones de la Universidad Pedagógica y Tecnológica de Colombia.

\section{Referencias}

Abdel, G., \& Romo, D. (2004). Sobre el concepto de competitividad. Documentos de trabajo en estudios de competitividad. Instituto Tecnológico Autónomo de México, ITAM. Centro de Estudios de Competitividad.

Augier, M., \& Teece, D. (2005). An Economics perspective on intellectual capital. Perspectives on Intelectual Capital, 3-27. DOI: 10.1016/B978-07506-7799-8.50006-1.

Bonet, L. (1993). La gerencia del talento humano en el siglo XXI. Publicaciones ICESI, 48, 37-43.

Cabrera, A., López, P., \& Ramírez, C. (2011). La competitividad empresarial: un marco conceptual para su estudio. Documentos de investigación, 4. Universidad Central. Recuperado de: http://www.ucentral.edu.co/sites/publicaciones/i mages/stories/subportal_publicaciones/publicac iones/Documentos-Administracion4.pdf.

Comunidad Andina de Naciones. (2009). Manual Técnico de Estadísticas Estructurales e Indicadores Básicos de Productividad y Competitividad. Resolución 1261 de 2009. Recuperado de: intranet.comunidadandina.org/Documentos/Res oluciones/RESo1261.doc.

Conpes 3527. (2008). Política nacional de competitividad y productividad. Alta Consejería Presidencial para la Competitividad y la Productividad, Ministerio de Comercio, Industria y Turismo, Consejo Privado de Competitividad. Recuperado de: file:///C:/Users/user/Downloads/ Conpes3527.pdf.

Costa, M. T., Duch, N., \& Lladós, J. (2004). Determinantes de la innovación y efectos sobre la competitividad: el caso de las empresas textiles. Document de treball 2000/4. Institut d'Economia de Barcelona. 1-25. Recuperado de: file:///C:/ Users/user/Downloads/DialnetDeterminantesDe LalnnovacionYEfectosSobreLaCompetit-2332 700.pdf.

CREPIB. (2010). Boyacá, Agroindustria productiva y competitiva. Boletines. Recuperado de: http://www.crepib.org.co.

Departamento Nacional de Planeacion. (2007). Agenda interna para la productividad y competitividad. Documento Regional Boyacá. Departamento Nacional de Planeación.

Echeverri, J., \& Escobar, A. (2004). Los determinantes de la competitividad en Colombia. Bogota D.C:Universidad de los Andes.

Esser, K., Hillebrand, W., \& Messner, D. (1999). Competitividad sistémica: nuevo desafío para las empresas y la política. Revista de la CEPAL, 59, 3952.

Fonnegra, N., \& González, N. (2006). Como hacer de las personas una ventaja competitiva: El Recurso Humano Como Un Factor Determinante Dentro De La Competitividad De La Organización. Universidad de La Sabana. Recuperado de: http://intellectum.unisabana.edu.co:8080/jspui/b itstream/10818/4486/1/131055.pdf.

Franco, I. (2009). Estudio de percepción del INVIMA. Instituto nacional de vigilancia de medicamentos y alimentos. 
García, B., \& Espino, L. (2011). Los recursos humanos como factor de competitividad en la PyME. 429-442. Universidad Autónoma del Estado de Hidalgo. Recuperado de: http://www.uaeh. edu.mx/investigacion/productos/4845/los_rec_h umanos_como_factor.pdf.

Grant, R. (1996). Toward a knowledge-based theory of the firm. Strategic Management Journal, $17,109-122$.

Lipovatz, D., Mandaraka, M., \& Mourelatos. (2000). Multivariate analysis for the assessment of factors affecting industrial competitiveness: The case of Greek food and beverage industries. Applied Stochastic Models in Business and Industry, 16(2), 85-98.

Martínez, C. (2012). Estadística y muestreo. Bogotá:Ecoe.

Nonaka, I., \& Takeuch, H. (1995). The Knowledge creating company: How Japanese companies create the dynamics of innovation. New York: Oxford University Press.
Porter, M. (1990). The competitive advantage of nations. New York: Free Press.

Sala-i-Martin, X., Blanke, J., Bilbao-Osorio, B., Browne, C., Crotti, R., Drzeniek, M., Findanza, B., Geiger, T., Gutknecht, T., Ko, C., \& Hanouz, M. (2012). The global competitiveness index 2012-2013. Strengthening recovery by raising productivity. World economic forum. Recuperado de: http://www3.weforum.org/docs/WEF_GlobalCo mpetitivenessReport_2012-13.pdf.

Teece, D. (1998). Capturing value from knowledge assets. The new economy, markets for know-how, and intangible assets. California Management Review, 40(3), 55.

Tsai, W. (2001). Knowledge transfer in intraorganizational networks: effects of networks position and absorptive capacity on business unit innovation and performance. Academy of Management Journal, 44(5), 996-1004. 